\title{
The Status and Influence of Zhucheng Guqin in the Development of Chinese Guqin Art
}

\author{
Xiaopeng Sun \\ Binzhou Polytechnic \\ Binzhou, China
}

\begin{abstract}
Guqin art has an extensive influence in Chinese musical, aesthetic, social and cultural, and ideological history. It is one of main representatives of Chinese ancient spiritual culture in music, and a valuable heritage of Chinese nation. Shandong Zhucheng Guqin School has about 200 years of history. It has an important role in the inheritance of folk culture. On the basis of the collection of the development of guqin schools and the inheritance of guqin culture in the history, the paper has combined the ontological study of Shandong Zhucheng Guqin and analyzed the significance and influence of Zhucheng Guqin on Chinese guqin art, and made an expectation on the development of Zhucheng Guqin.
\end{abstract}

Keywords-Guqin culture; Zhucheng guqin; ontological study; development prospect

\section{The DeVelopment Of GuQIn SCHOOLS In ThE HISTORY}

The formation of guqin schools is dependent on mature concepts of guqin masters. The qin theoretical view of thought has a close relationship with traditional Confucianism and Taoism. The qin theoretical view of thought is the embodiment of Confucianism and Taoism in qin theory. In the long development art road, the ultimate reflection of Chinese guqin music culture is a mental state combining an aesthetic art with philosophical ideas. It has an inseparable relation with the earliest Taoist and Confucian culture. Its expressive force is complementary to the two major philosophical thoughts. Use art to express philosophical thought and people's spiritual pursuit, which is also a spiritual characteristic for Taoism and Confucianism to express people's spiritual pursuit [1].

Since there were written records in the history, there were musical instruments. These musical instruments, including the earliest beat percussion instruments and plucked instruments and modern synthetic electronic instruments, are an important part of human culture. Guqin is one of the earliest popular plucked instruments. According to Historical Records, there was Guqin as early as the Yao and Shun period. It was very popular in the Spring and Autumn Period. It has more than 3000 years of history. Guqin is one of the most representative musical instruments in ancient China. Guqin is very different from Western percussion or wind instruments. Western organ is lively, jumping and flexible, with rich emotions and high freedom. Guqin is fresh and elegant, and it often implies the lofty and unyielding living attitude of ancient scholars. With a unique style and rich connotations, it has a unique qin temperament, notation method, playing method and aesthetic standards, forming a complete set of unique system.

Qin culture in the ancient feudal society is a symbol of nobility and gifted scholars and beautiful ladies. The broad and profound content has a close link with philosophy, history and literature. It is a representative of Chinese traditional music [2]. According to the records of ancient qin masters, the qin culture can be traced back to about 3000 or 4000 BC. In the Spring and Autumn and the Warring States Period, the performance of qin has been very common in the countries. There were many legends about qin in this period. For example, Boya empathized and composed Shuixian Cao, and composed High Mountain and Flowing Water in seeking friends. Zhong Yi kept homeland in mind and touched the marquis of Jin State. Confucius toured various countries and composed Orchid. They are all well-known stories. During this period, a number of famous qin masters emerged, such as, Boya, Confucius, Shi Kuang and Shi Xiang. At that time, the qin culture has already had a considerable development. The recorded famous qin masters at that time were Shi Juan of Wei State, Shi Kuang of Jin State, Shi Wen of Zheng State, Shi Xiang of Lu State and etc. The recorded qin songs were High Mountain, Flowing Water, Zhichaofei, Sunny Spring and White Snow, and they are still played today [3]. To the Han and Jin Dynasties, the qin culture has been very prosperous. Guqin culture has had a great development. In the Sui and Tang Dynasties, with the political and economic prosperity, the qin culture has also been prosperously developed. In the Tang and Song Dynasties, it was the development peak of Chinese culture. The guqin art has also been considerably developed in the contention of a hundred schools of thought, and further developed in the Yuan, Ming and Qing Dynasties. In different historical periods, there were qin masterpieces spread up to now.

Chinese music has more than three thousand years of history, but in fact from the ancient times to the end of the Tang Dynasty, the direct sound materials (music) were extremely poor. Therefore, the study of ancient Chinese music history can only be started from some indirect materials (such as literature, musical instruments, carving, painting, etc.). Guqin is one of Chinese musical instruments with many sound materials, so it is really a nonnegligible music treasure for us to understand ancient Chinese traditional music styles and features. 


\section{THE HISTORICAL INHERITANCE OF GUQIN CULTURE}

In the long history of qin culture, the historical inheritance of guqin culture is mainly manifested in two aspects, the evolution of guqin schools and changes of Qin-making technologies.

\section{A. The Evolution of Guqin Schools}

The guqin art is developed in the succession of teachings from a master to his disciples, so its playing styles are much diversified. It is an inevitable result of cultural development in the contention of a hundred schools of thought, and also a power to drive the development of literature and art. Due to different regions, schools and historical periods and social, political and economic situations, the expressed art concepts of guqin culture are also different, and thus forming different performing styles. The differences on understanding the art and expression are reflected in artistic concepts and performing styles. In its development, guqin schools often evolved and changed. Relying on their regional development characteristics, it formed different schools with different art concepts and performing styles. For example, there were Shushan Qin School, formed in the Han Dynasty, with Sima Xiangru, Yang Xiong, Zhu Geliang and Jiang Wei as representative, and Zhulin School in the early Jin Dynasty. They had their own characteristics, just like the words of Zhao Yeli of Sui and Tang Dynasties: "the music of Wu School is fresh and mellow, just like the bicker of river, continuous and long. The music of Shu School is urgent and impetuous, just like stirring spindrifts and running thunder"[4]. The recent schools mostly are classified based on their regions, such as, Zhe School, Songjiang School, Jinling School, Wu School, Zhongzhou School, Min School, Lingnan School, Chuan School, Jiuyi School, Zhucheng School, Guangling School, Yushan School, Shaoxing School, Mei'an School and so on.

Guangling School is centered on Jiangsu Yangzhou. Xu Changyu is the founder. The famous qin masters are $\mathrm{Xu}$ Changyu, Xu Qi, Wu Hong, Qin Weihan, Shi Kongchen and so on. The important scores include Qin Score of Chengjian House, Qin Score of Wuzhi House, Qin Score of Ziyuan House, Qin Score of Jiao'an Temple, and Qin Score of Kumu Temple. The representative songs are Longxiang Cao, Plum Blossom Melody, Wild Geese on the Beach, Xiaoxiang Shuiyun, and Song of Guangling. The artistic style is just, free, easy and distant.

Yushan School, also known as Changshu School, is centered on Jiangsu Changshu. It was the most influential school in the end of Ming Dynasty and in the early of Qing Dynasty. The famous qin masters are Yan Tianchi, Xu Qingshan, Xia Pu, etc. The important scores include Qin Score of Songxian Pavilion, Qin Score of Dahuan Pavilion, and so on. The representative songs are Anchored on Autumn River, Tune for a Peaceful Night, Xiaoxiang Shuiyun. The artistic style is fresh and elegant.

Zhe School is centered on Lin'an of Southern Song Dynasty. Its qin art has affected the Yuan Dynasty and Ming Dynasty. The famous qin masters are Guo Chuwang, Liu Zhifang, Xu Tianmin, Mao Minzhong and etc. The important sources are Qin Score of Zixia Cave, Score of Qin Cao, Qin
Shu, Xiawai Qin Score, Qin Theory Dictum, Meixuewo Qin Score, Wugang Qin Score, Qin Score of Xingzhuang Taiyin and so on. The representative songs are Song of Fisherman, Song of Woodman, Xiaoxiang Shuiyun, Eighteen Beats of Hujia and so on. The artistic style is fluent and gentle.

Shu School is also known as Chuan School. The famous qin masters are Zhang Kongshan, Tang Yiming, Gu Yucheng, Chen Yunhe, Gu Meigeng, Yu Shaoze, Yu Bosun, Wang Dehua and etc. The important scores were Qin Score of Tianwen Pavilion, Music Score of Baiping House. The modern music scores are Shayan Qin Compilation, Qin Score of Caotang House and etc. The representative works are Flowing Water, Evening Song of Drunken Fisherman, Confucius Duyi, $P u$ 'an Incantation and etc. The artistic style is impatient, bold and magnificent.

Mei' an School is a new rising school. It can be traced back to the Qing Dynasty. The founder is Wang Binglu and $\mathrm{Xu}$ Lisun. The well-known qin masters were Wang Yanqing, $\mathrm{Xu}$ Lisun, Shao Dasun, Wang Yongchang, etc. The important scores are Qin Score of Longyin Pavilion, Qin Score of Mei 'an Tempe and so on. The representative works are Wild Geese on the Beach, Changmenyuan, Guanshan Moon, Anchored on Autumn River, Daoyi and so on. The artistic style is fluent, beautiful and lingering, with large trill.

Lingnan School is originated in the period under the reign of Daogang Emperor of Qing Dynasty. The famous qin masters are Huang Jingxing, Li Baoguang, Zheng Jianhou, Yang Xinlun and etc. The important music scores were Gugang Qin Score, Qin Score of Wuxueshan House and etc. The representative works are Flowing Fountain in Green Cave, Conversation of Fisherman and Woodman, Recalling Antiquity, Jade Tree, Innocent Seagulls, Double Crane Listening Fountain and so on. The artistic style is tender and elegant.

Min School is a modern qin school. The representative Zhu Fengjie composed Qin Score of Yugu Pavilion. The following scholar Zhang He compiled Introduction to Qin Theory; and Chen Shiji compiled Foundation of Qin Theory.

Zhucheng School is an important school in the development of guqin under the background of the contention of a hundred schools of thought, and the most influential school in the country. It is centered on Shandong Zhucheng. The famous qin masters were Wang Puchang, Wang Yumen, Wang Lu and etc. The important music scores were Qin Score of Tongyinshan House, Qinpu Zhenglv and so on. The representative works are Changmenyuan, Yangguan Sandie, Guanshan Moon and etc. The artistic style generally is strong but pliable, thick but sparse, full but empty, playing at a stretch. The tone is mellow and deep, with airy beauty. The music is tight and continuous, circulatory and remote, powerful and splendid.

\section{B. Changes of Qin-Making Technologies}

The length of guqin generally is three Chi six Cun and five Fen, symbolizing one year of time. The width of guqin generally is four Cun, symbolizing four seasons in one year. There are twelve months in one year. If add a leap month, 
there will be thirteen months. This number is just the total number of "hui" in musical scale of guqin.

The material of qin body is the main factor influencing guqin's timbre. Face plate of guqin generally is made of paulownia wood or cedarwood. The wood shall be dried, otherwise it will crack up and become warped, and the timbre will go bad. It takes a long time for wood to be dried naturally. So, qin makers often collect all kinds of ancient woods (coffin wood of ancient tombs or beam columns of ancient house) to make qin. Face plate and bottom plate of guqin are glued with fish glue.

First, paint a layer of gray paint on the face plate of guqin, which can protect the soft face of qin. The most commonly used gray paint is antler ash mixed with cornu cervi degelatinatum and raw lacquer. Paint a layer of Chinese lacquer (raw lacquer) on the surface of guqin. Mostly, use black lacquer, and seldom use red, brown or purple lacquer. Without completely dry, polish the surface of guqin with water-dipped oilstone. Other accessories, such as Yueshan, Chenglu, Longyin, Yanzu, Qinzhen, are made of hard wood or jade. There are 13 Hui, made of mother-of-pearl inlay or jade.

Guqin, also known as "seven-stringed civil and military qin", is a musical instrument full of cultural connotations. Poet of Tang Dynasty Liu Changqing wrote: “泠泠七弦上, 静听 松风寒”. “七弦” represents guqin, and “泠泠” represents the tone of guqin.

In Chinese traditional culture, people believe that there are five stars in the sky and five elements on the ground. There are five notes in the world. Therefore, Shen Nong created the first qin with paulownia and rope yarn. At that time, there were only five strings, including Gong, Shang, Jiao, Hui and Yu. The five strings respectively symbolize gold, wood, water, fire and earth. Later, King Wen of Zhou State added one string in order to mourn his died son Bo Yikao. King Wu of Zhou State also added one string in order to increase morale. Guqin was thus generated. It has 5000 years of history from today. Sevenstringed qin, Chinese guqin, has deep and magnificent tune, stable and beautiful shape. It contains infinite secrets, vibrating thousand years of literacy excellence[5].

Guqin is beautiful in shape and various in type, including Zhongni Type, Fuxi Type, Luoxia Type, Lianzhu Type, Shennong Type, Jiling Type, Jiaoye Type and so on. They are different from each other. The qin-making technologies are also different in different periods. Each qin maker has his style. The qin-making directories in each generation were Taiyin Collection in the Northern Song Dynasty, Qinyuan Selections in the Song Dynasty, Fengxuan Xuanpin in the Ming Dynasty and so on. The famous qin makers were Lei Clan, Guo Liang and Zhang Yue in the Tang Dynasty; Zhu Renji, Ma Xiliang, Seng Renzhi, Jin Zhou, Jin Gonglu and Chen Hengdao in the Song Dynasty; Zhu Zhiyuan in the Yuan Dynasty and Zhang Jingxiu in the Ming Dynasty, and so on.

\section{The OntologicAl ReseARCH OF ZHUCHENG GuQIN}

\section{A. The Characteristics of Shandong Zhucheng Guqin}

Since the Qing Dynasty, the tune-establishing system of guqin is generally classified into two types. One takes the third string as Gong and uses five notes (Gong, Shang, Jiao, Hui and $\mathrm{Yu}$ ) to name tune. The "do" tune of the third string is named Gong; the "re" tune of the third string is named Shang; the "mi" tone of the third string is named Jiao; the "sol" tune of the third string is named Hui; the "la" tune of the third string is named $\mathrm{Yu}$. The other takes the first string as Gong and uses "Jun" to name tune. The "do" tune of the first string is named Huangzhong Yun; the "re" tune of the first string is named Wushe Yun; the "mi" tune of the first string is named Yize Yun; the "sol" tune of the first string is named Zhongni Yun; the "la" tune of the first string is named Jiazhong Yun. The tune system of Zhongcheng School has combined the two types. It takes the third string as Gong and uses the Lvlv tunenaming method. The tune with the third string as Gong is named Huangzhong Tune ( $\mathrm{F}$ tune). The pitch names respectively are sol, la, do, re, mi, sol, la. The tune with the first string as Gong is named Linzhong Tune (C tune). The pitch names respectively are do, re, mi, sol, la, do, re. The tune with the second string as Gong is named as Wushe Tune (bE tune). The pitch names respectively are la, do, re, mi, la, do. The tune with the fourth string as Gong is named Taicu Tune (G tune). The pitch names respective are mi, sol, la, do, re, mi, sol. The tune with fifth string as Gong is named Zhongni Tune (bB tune). The pitch names respectively are re, mi, sol, la, do, re, mi.

Zhucheng Guqin has subtle and implied sound quality. The performance is plain and simple. The performing skills are fluent, implied and general. It emphasizes internal expressive force, rather than external magnificence. The music is pressing or easing, hard or soft. The rhythm of a song is fixed with uniform standard. Several guqin can play at same time. It is easy to divide rhythm and equipped with numbered music notation. The performing skill of right hand shall be rigid and rigorous, clear and agile with strong strength.

\section{B. The Representative Works of Zhucheng Guqin}

The representative works of Zhucheng School are Wild Geese on the Beach, Asking the Heaven, Daoyi, Flowing Water and Changmenyuan. Other schools don't often play the song of Daoyi, and only Zhucheng School still teaches this song today. The most widely spread songs are Wild Geese on the Beach and Changyuanmen. Almost every school can play them. In these songs, the three songs, including Guanshan Moon, Changmenyuan, Autumn Wind, are unique in the qin scores of Zhucheng School. There is no such record in the music score of other schools.

\section{The Guqin Scores of Zhucheng School}

Qinpu Zhenglv is collected by Qin Master Wang Lengquan. It is a manuscript. The original one has been lost. In the spring of 1961, in Zhucheng Agriculture University, an incomplete manuscript was found from Zhao Hao. And in the winter of same year, the second-hand manuscript of Qinpu Zhenglv, transcribed by Wang Lengquan, was found from Zhan 
Chengqiu. Since then, it was combined together. The opening is a guide for use and an introduction to tunes and strings. The recorded songs are Wild Geese at the Beach, Song of Fisherman, Autumn Swan, Innocent Seagulls, Tune for a Peaceful Night, Changmenyuan, Xiaoxiang Shuiyun, Dancing Leaf with Wind, Jingguan Yin, Shanju Yin, Saishanghong, Hujia, Shuixian Cao and so on. According to the record, Changmenyuan was originated from Qin Score of Tongyinshan House; the seven songs, such as, Jingguan Yin, were originated from Qin Score of Wuzhi House. Wang Lengquan further corrected the position of Hui. In the song of Autumn Swan, individual paragraphs were merged in the tune-establishing system of Zhucheng School.

Qin Score of Tongyinshan House is passed by three generations of guqin family, Wang Jifu, Wang Xinyuan and Wang Xiunan. The songs include Daoyi, Asking the Heaven, Wild Geese on the Beach, Tune for a Peaceful Night, Flowing Water, Pu'an Incantation, Autumn Wind, Changyuanmen, Anchored on Autumn River, Flowing Fountain from Stone, Cantongqi, Duyi, Chunguiyuan, Qiusaiyin, Yangguan Sandie, with a total of 15 songs. It also contains left and right hand fingering, score reading method and string regulating methods. These are relatively primitive songs. There are also some other songs in other qin score books, which all come from it. After liberation, they were sorted and printed by modern qin master couple Zhang Yujin and Wang Fengxiang. The valuable point is that there is a line of numbered musical score on the "jianzi score" of guqin with right and left hand fingering but accurate rhythm, and it retains the true color of Zhucheng Guqin.

Qin Score of Yuhe Pavilion was edited and revised by Qin Master Wang Xinkui, with a total of eight volumes. It is a manuscript. The original one was burned by fire. The songs are Wild Geese on the Beach, Plum Blossom Melody, Changmenyuan, Xiaxianyou, Hangong Qiuyue, Clear Fountain from Stone, Saishanghong, Hujia, Song of Fisherman, Song of Woodman, Anchored on Autumn River, Peilan, Autumn Swan, Conversation of Fisherman and Woodman, Hewu Dongtian, Fengqiuhuang, Qiusai Yin, Tune for a Peaceful Night, Cangwuyuan, Chunguiyuan, Chunxiao Yin, with a total of 13 songs.

Mei'an Qin Score is passed down by Qin Master Wang Yanqing. When Mr. Wang died, it was edited by Xu Lisun and Shao Dasu. It was published in 1931, with a total of two volumes. The first volume writes music theory, fingering and chord. The second volume has 14 songs, including Anchored on Autumn River, Long Autumn Night, Daoyi, Guanshan Moon, Yulou Chunxiao, Autumn Wind, Jile Yin, Xiaxianyou, Fengqiuhuang, Wind and Thunder, Changmenyuan, Wild Geese on the Beach, Shitanzhang, and Asking the Heaven. Three songs of them were originated from Qin Score of Tongyinshan House, and modified by Wang Yanqing afterwards.

\section{THE INFLUENCE AND SIGNIFICANCE OF ZHUCHENG GUQIN ON CHINESE GUQIN ART}

\section{A. The Significance of Zhucheng Guqin on Chinese Guqin Art}

Shandong Zhucheng Guqin has experienced continuous exploration, exchange, absorption and development of several generations of qin masters, gradually forming a guqin school with unique characteristics, style, skill and certain songs. It is a peculiar guqin art school in modern qin circle in China, and is one of famous qin schools in China. It has been developed up to now. There are many guqin inheritors of Zhucheng School in Shandong, Jiangsu, Henan, Hubei, Zhejiang, Shaanxi, Hebei, Guangdong, Sichuan and Tianjin. Qin masters of Zhucheng School have great influence on society with their noble personality and exquisite Qin art. They have won high attention of many well-known scholars, educators and politicians, which further expands the influence of Zhucheng Guqin. Chinese guqin circle is mystery and conservative, with many sacred rules, so it drifts away from real life. Qin masters of Zhucheng School disregard those rules, and extensively draw nutrition from vibrant folk music. Their behavior has affected other qin schools, so as to bring new changes to the inheritance and development of Chinese guqin. Guqin art has renewed a brilliant luster in the land of China.

\section{B. The Influence of Shandong Zhucheng Guqin in the Development of Chinese Guqin Art}

Zhucheng Guqin Art has gone through more than 200 years of development, which has a far-reaching influence on social culture and era thought. It is an important music art form in ancient China, and a valuable heritage of Chinese nation. It helps cultivate the mind and edify sentiment to learn and play guqin, so more lovers can participate in it. In spite of skills, people can enjoy the beauty of its spiritual treasure. People can sublimate themselves through studying and learning guqin. We want to see that the scores of these songs silent all these years can be printed out, the traditional culture can be understood and valued, the research of guqin theory can be more standard and deepened, and there are more academics results for people's exploration. What's more, the noble character of guqin can be integrated with the ideological character of our countrymen. So, the musical instrument with traditional Chinese cultural characteristics can serve the people, convey immortal Chinese spirit to the world and enable the outstanding traditional culture treasure to pass on forever.

China traditional culture is broad and profound, which is a charm of Chinese ancient culture. From the 200 years of development history of guqin culture, we can see that the art charm of Zhucheng Guqin culture is very profound. It has trails and opportunities in its development, and never comes to a halt in continuous infusion of the history cultural heritage and changes of humanistic essence. In modern trend, Zhucheng Guqin faces more opportunities and challenges, which is the necessary way in the development of our folk culture. Change in the development and advance in the changes, which is a general rule in the inheritance and development of folk culture. It is a new idea for the development of Chinese traditional music culture to study the 
development path of Zhucheng Guqin art, promote the service of folk art for people, vigorously cultivate talents and promote the inheritance and development of guqin culture in modern developmental mode. It is a mutual orientation of modern guqin art to develop the national spirit with innovative spirit and carry forward the national culture with the spirit as the Foolish Old Man, who removed the mountains.

\section{The DeVelopment Prospects of ZHUCHENG GuQIN}

Under the guidance of a healthy social atmosphere, the development prospect of Zhucheng Guqin will also be very optimistic. Our modern society has stable politics and developmental economy, and people's living standards continue to improve. It is bound to arouse people's enthusiasm to pursue spiritual enjoyment. Guqin art, like other folk music culture, also is sought after by all sectors of society. There are more and more people learning guqin. People pay more and more attention on guqin art, and their quality on folk culture is also improved gradually. It is a basic requirement for people to cultivate the mind with music, which is the soil for the development of Zhucheng Guqin. In recent years, excellent talents of Zhucheng School come out in succession. Many qin masters are active in the Zhucheng Guqin art stage. They have made a great contribution, and we place high hopes on them. In near future, Zhucheng Guqin art will once again create brilliance like the contention of a hundred scholars of thought. It will surpass the past and run toward a higher artistic peak.

\section{ACKNOWLEDGMENT}

We would like to thank all reviewers and editors for their valuable comments and suggestions during the review process.

\section{REFERENCES}

[1] Liu Hui, Liu Zhong. Aesthetic Characteristics of Chinese Music for Literatus. Xi'an: Journal of Lanzhou University, 2003.

[2] Ma Huimin. Core Concepts of Guqin and Chinese Ancient Aesthetic Thoughts. Xinjiang: Journal of Kashi Teacher's College, 2006.

[3] Edited by Beijing Guqin Research Association. Guqin Music Collections. Beijing: People's Music Publishing House, 1972.

[4] Feng Guangyu. Guqin Music and Modern Culture. Beijing: People's Music Publishing House, 1995.

[5] Licun. Rhythm of Guqin Jianzi Score. Shaanxi: Journal of Xi'an Conservatory of Music, 2008. 